No se encontraron diferencias significativas entre las medidas antropométricas iniciales y finales. Se observó un mejoramiento significativo en los parámetros que reflejaban el estado físico de las participantes en el grupo que realizaba ejercicios, mientras que en el grupo testigo dichos paramétros permanecieron sin cambio o empeoraron. Los valores de densitometría ósea se mantuvieron estables en el grupo que realizaba ejercicios, pero en el grupo testigo se observó una disminución significativa en la región lumbar de la columna vertebral y en la zona proximal del fémur, con valores significativamente inferiores a los del grupo que realizaba ejercicios al final del estudio.

El programa de ejercicios realizado redujo significativamente las concentraciones de colesterol total en $5 \%$ y de triglicéridos en $14 \%$, mientras que en el grupo testigo estos parámetros aumentaron en $4 \%$ y $23 \%$, respectivamente. De esta manera al finalizar el programa de ejercicios, los valores de colesterol total y triglicéridos del grupo de mujeres que realizaban ejercicios fueron significativamente menores que los del grupo testigo. No se observaron diferencias en los valores de colesterol ligado a lipoproteínas de alta densidad entre ambos grupos.

Se observó una tendencia descendente de los síntomas vasomotores; sin embargo, solo se encontraron diferencias significativas entre el grupo que realizaba ejercicios y el grupo testigo en relación con el estado de ánimo y el insomnio.

La intensidad y la frecuencia del dolor entre las participantes que realizaban ejercicios disminuyeron significativamente, lo que provocó que se encontraran diferencias significativas entre los dos grupos al final del estudio. A pesar de los ejercicios intensos, no se informaron cambios con relación al estado de las principales articulaciones.

Los marcadores séricos del recambio óseo no mostraron variaciones significativas en ninguno de los dos grupos. La densidad mineral ósea disminuyó $2,3 \%$ en el grupo testigo, mientras que en las participantes del grupo que realizaba ejercicios permaneció estable. En la región lumbar de la columna vertebral, los ejercicios permitieron detener el avance de la reducción de la densidad mineral ósea trabecular y aumentó significativamente la fortaleza de la corteza, gracias al aumento del grosor del hueso o al incremento de su densidad mineral ósea. Se observó una tendencia similar en la zona proximal del fémur, aunque esta fue menos pronunciada que en la columna vertebral.

La eficiencia del sistema cardiovascular, según los valores del consumo máximo de oxígeno, aumentó significativamente en las participantes en el grupo de ejercicios $(+12,4 \%)$ y disminuyó, aunque de manera no significativa $(-2,3 \%)$, en el grupo testigo.
Este trabajo demuestra que un programa de ejercicios generales intensos dirigidos especialmente a mejorar la densidad ósea en mujeres posmenopáusicas puede evitar la pérdida ósea y mejorar significativamente la fortaleza y la resistencia, además de reducir el dolor de espalda y las concentraciones de lípidos en sangre en este grupo de mujeres. (Kemmler W, Lauber D, Weineck J, Hensen J, Kalender W, Engelke K. Benefits of 2 years of intense exercise on bone density, physical fitness, and blood lipids in early postmenopausal osteopenic women. Results of the Erlangen Fitness Osteoporosis Prevention Study (EFOPS). Arch Intern Med. 2004;164:1084-91.)

\section{El liraglutide permite controlar la glucemia sin aumento de peso en pacientes con diabetes tipo 2}

La diabetes tipo 2 es una enfermedad caracterizada por la resistencia a la insulina y la presencia de células $\beta$ deficientes, y está asociada con la hiperglucagonemia, el aumento de la producción de glucosa en el hígado y la obesidad. Los pacientes con diabetes tipo 2 segregan menos cantidad de péptido 1 similar al glucagón (PG-1) durante las comidas. Diversos estudios han demostrado que la hormona PG-1 estimula la secreción de insulina, inhibe la secreción de glucagón en dependencia de los niveles de glucosa y retrasa el vaciamiento gástrico, lo que provoca la disminución del apetito y la pérdida de peso de los pacientes. Sin embargo, la vida media del PG-1 natural es muy corta (alrededor de 1 minuto) debido a la acción de la enzima dipeptidil peptidasa I. Además, se ha demostrado que para ejercer su acción, el PG-1 debe estar presente permanentemente en el torrente sanguíneo. A pesar de estos inconvenientes, esta hormona puede servir para el tratamiento de la diabetes tipo 2 .

El liraglutide es un análogo acilado del PG-1, de acción prolongada, que actúa como antagonista de los receptores de esta hormona. Estudios realizados en animales y humanos han demostrado el efecto positivo de este fármaco en la reducción de las concentraciones de glucosa en sangre y la seguridad de su empleo. La vida media del liraglutide después de una dosis única o de múltiples dosis es de aproximadamente 12 horas, tanto en personas sanas como en pacientes con diabetes tipo 2 . El régimen terapéutico se basa en una inyección diaria.

El objetivo de este estudio fue determinar la eficacia y la seguridad del liraglutide después de 12 semanas de tratamiento en pacientes con diabetes tipo 2. Para ello se realizó un ensayo clínico multicéntrico aleatorizado con doble enmascaramiento. A los pacientes se les pidió mantener durante la realización del ensayo la dieta acostumbrada y sus 
programas de ejercicios habituales. Después de un reposo farmacológico de cuatro semanas durante el cual se suspendieron los tratamientos con hipoglucemiantes orales, los pacientes se asignaron a uno de cinco grupos que recibieron diferentes dosis de liraglutide $(0,045,0,225,0,45,0,60$ ó $0,75 \mathrm{mg})$ o placebo. También se estableció un grupo testigo que recibió de 1 a $4 \mathrm{mg}$ de sulfonilurea (glimepiride). El ensayo se llevó a cabo entre diciembre de 2000 y octubre de 2001 en los países escandinavos y en el Reino Unido.

En total se evaluaron 311 pacientes con diabetes tipo 2 de uno $u$ otro sexo, de 30 años de edad o más y con índice de masa corporal de $40 \mathrm{~kg} / \mathrm{m}^{2}$ o menos. Todos recibían tratamiento con algún hipoglucemiante oral (con valores de $\mathrm{HbA}_{1 c} \leq 9,5 \%$ ) o mantenían una dieta de control (con valores de $\mathrm{HbA}_{1 \mathrm{c}}$ de 7,5 a 10,0\%). Se excluyó a los pacientes con enfermedades hepáticas o renales, insuficiencia cardíaca, angina de pecho inestable, antecedentes de infarto del miocardio en los 12 meses anteriores al estudio, tratamiento concomitante con tiazolidinedionas o algún otro medicamento en investigación, $u$ otra enfermedad que pudiera incidir en la diabetes del paciente o en sus posibilidades de participar en el ensayo hasta el final. Se excluyó también a las mujeres embarazadas, las que estaban amantando a sus hijos pequeños y las que no empleaban algún anticonceptivo adecuado. Los pacientes que durante el ensayo presentaron valores de glucosa plasmática en ayunas superiores a 15 $\mathrm{mmol} / \mathrm{L}$ se retiraron del ensayo.

Para el análisis final se tomaron en cuenta 190 pacientes que se estudiaron en siete ocasiones: $\mathrm{du}-$ rante el tamizaje inicial; al inicio del tratamiento; después de 1, 4, 8 y 12 semanas de tratamiento; y durante el seguimiento. No se encontraron diferencias significativas entre los diferentes grupos de pacientes en el momento del inicio del ensayo.

Este estudio fue el primero en demostrar la mejoría sostenida en el control de la glucemia después del tratamiento prolongado con liraglutide con una dosis diaria y esta mejoría pudo observarse desde la primera semana de tratamiento. Después de 12 semanas, los valores de $\mathrm{HbA}_{1 \mathrm{c}}$ disminuyeron en todos los grupos, excepto en el correspondiente a la menor dosis de liraglutide y esta reducción fue estadísticamente significativa entre los grupos que emplearon las dos dosis mayores con relación al grupo que recibió placebo. Con estas dosis, el efecto del liraglutide sobre los valores de $\mathrm{HbA}_{1 \mathrm{c}}$ fue similar al obtenido con glimepiride. Este efecto aumentó a medida que avanzó el tratamiento. La mayor disminución en los valores de $\mathrm{HbA}_{1 \mathrm{c}}$ se observó al final de las 12 semanas de tratamiento con la mayor dosis de liraglutide, aunque al parecer esta tendencia podía haber continuado si no se hubiera interrum- pido el tratamiento. Se observó que $59 \%$ de los pacientes que completaron el ensayo en los dos grupos con mayores dosis de liraglutide alcanzaron valores de $\mathrm{HbA}_{1 \mathrm{c}} \leq 7 \%$ después de 12 semanas.

Las concentraciones de glucosa sérica en ayunas disminuyeron durante el ensayo en la mayoría de los grupos de tratamiento, con reducciones significativas entre los grupos que emplearon dosis de $0,225,0,60$ y 0,75 mg de liraglutide en comparación con el grupo que recibió placebo. El efecto de la mayor dosis de liraglutide fue similar al observado en el grupo que recibió glimepiride. En los grupos tratados con liraglutide, los valores de glucosa sérica en ayunas disminuyeron a partir de la primera semana del ensayo.

En promedio, la función de las células $\beta$ (según los valores de insulina y glucosa en ayunas) fue significativamente mejor después de las 12 semanas del ensayo en el grupo tratado con $0,75 \mathrm{mg}$ de liraglutide que en el grupo que recibió placebo. En cuanto a la resistencia a la insulina, no se observaron diferencias significativas entre los tres tratamientos (liraglutide, glimepiride y placebo). Además, la disminución de la relación proinsulina:insulina fue estadísticamente mejor en el grupo que recibió $0,75 \mathrm{mg}$ de liraglutide en comparación con el que recibió placebo. No se observaron diferencias estadísticamente significativas en cuanto a los valores de insulina en ayunas, de péptido $\mathrm{C}$ y de glucagón entre los grupos tratados con liraglutide y el que recibió placebo.

De los 135 pacientes que recibieron liraglutide, solo $1(0,7 \%)$ (del grupo que recibió $0,50 \mathrm{mg}$ ) sufrió un episodio menor de hiploglucemia (definida por valores de glucosa en sangre $<2,8 \mathrm{mmol} / \mathrm{L}$ ) y $7(5,2 \%)$ informaron síntomas de hipoglucemia. Esta proporción fue menor que la observada en el grupo de glimepiride $(N=26)$, en el que 4 pacientes $(5,4 \%)$ sufrieron episodios menores de hipoglucemia y $5(19,2 \%)$ informaron de haber tenido síntomas de hipoglucemia.

El peso corporal disminuyó en $1,2 \mathrm{~kg}$ en el grupo que recibió $0,45 \mathrm{mg}$ de liragluide en comparación con el grupo que recibió placebo $(P=0,0184)$. Esta observación respalda los hallazgos de que el PG-1 reduce el consumo de energía en los pacientes.

El número de pacientes que presentaron reacciones adversas (según la información espontánea ofrecida por los participantes) fue similar en todos los grupos que recibieron liraglutide o placebo (81 de $135[60 \%]$ frente a 16 de 29 [55\%], respectivamente), y fue menor en el grupo testigo que recibió glimepiride (9 de 26, 35\%). En general, las reacciones adversas informadas fueron leves o moderadas y en todos los casos se resolvieron completamente. Las reacciones adversas más frecuentes fueron dolor de cabeza y nauseas, que son las informadas habitualmente para el fármaco evaluado. No se de- 
tectaron anticuerpos contra el medicamento. Los resultados de laboratorio no mostraron alteraciones en los signos vitales o electrocardiográficos que afectaran a los pacientes.

En conclusión, el liraglutide permitió reducir las concentraciones de glucosa en sangre en pacientes con diabetes tipo 2 sin provocar el aumento de peso o del riesgo de hipoglucemia. (Madsbad S, Schmitz O, Ranstam J, Jakobsen G, Matthews DR, et al. Improved glycemic control with no weight increase in patients with type 2 diabetes after oncedaily treatment with the long-acting glucagon-like peptide 1 analog liraglutide (NN2211). A 12-week, double-blind, randomized, controlled trial. Diabetes Care. 2004;27:1335-42.)

\section{Algoritmos para determinar el umbral de alerta ante epidemias de malaria}

Los sistemas sanitarios requieren de mecanismos que les permitan pronosticar el incremento inusual del número de casos de determinadas enfermedades. Esos sistemas son particularmente necesarios en regiones propensas a la aparición de epidemias, ya que permiten emprender acciones oportunas de control y mitigar el impacto de esas epidemias sobre la salud de la población.

Por lo general, la detección de un número inusual de casos de una enfermedad en un momento determinado puede ser un indicio de que una epidemia está por aparecer. Los sistemas de alerta epidemiológica se basan en algoritmos diseñados para calcular los valores umbrales (número de casos) que pueden indicar con mayor certeza y antelación la aparición de las epidemias.

Sin embargo, hasta el momento no se han desarrollado métodos que puedan evaluar los sistemas de detección temprana y su capacidad para generar alertas oportunas. La utilidad de estos sistemas de alerta depende de la sensibilidad y la especificidad con que permitan identificar las epidemias en sus fases más tempranas.

En el caso de la malaria, se ha demostrado la utilidad de realizar intervenciones oportunas - como la eliminación de larvas, la fumigación de los residuos domésticos y la administración masiva de medicamentos- para controlar las epidemias, pero no se conoce aún la forma de identificar el momento óptimo para emprender esas acciones, especialmente cuando los recursos son escasos.

En este trabajo se describe un método empleado para evaluar varios sistemas sencillos de detección temprana de epidemias de malaria, según su capacidad para brindar alertas sensibles, específicas y oportunas. Para ello se emplearon los datos semanales de casos confirmados de malaria en 10 localidades de Etiopía entre 1999 y 2000. Los datos se obtuvieron según las semanas etíopes (que pueden ser de 5 a 9 días), por lo que se normalizaron para el análisis.

Se analizaron cuatro algoritmos para el cálculo del umbral de alerta. En todos los casos, la alerta se establecía si el número semanal de casos excedía ese umbral durante dos semanas consecutivas, a fin de aumentar la especificidad del sistema de alerta, independientemente del algoritmo empleado.

Los algoritmos analizados se basaron en los percentiles semanales (según el número de casos informados en esa misma semana durante los años estudiados, excepto en el año en cuestión); la media semanal más su desviación estándar (la media semanal del número de casos más un número dado de desviaciones estándar); el porcentaje de muestras positivas (proporción de muestras positivas con relación al número de muestras analizadas); y la pendiente del número de casos positivos semanales en una escala logarítmica (el umbral se define según la pendiente del logaritmo natural del número de casos semanales normalizados).

Cada algoritmo se evaluó según el número de alertas que hubiera desencadenado y el número de casos que se hubieran podido prevenir (NCP) si se hubiera aplicado la alerta. El NCP se definió como una función del número de casos detectados entre la $2 .^{a}$ y la $8 .^{a}$ semanas posteriores a la alerta, o entre la 2. ${ }^{a}$ y la $24 .^{a}$ semanas, en dependencia de la duración de las medidas de intervención.

En el período evaluado se detectaron 687903 casos de malaria. En promedio, cada una de las 10 instalaciones sanitarias participantes (una por cada distrito) trató entre 11 y 19 casos de malaria diariamente y más de 300 casos diarios durante las temporadas de mayor transmisión.

Según el número de alertas desencadenadas y la proporción de NCP para cada umbral, los sistemas de alerta basados en los algoritmos estudiados hubieran podido prevenir un mayor número de casos que las alertas anuales aleatorias.

El umbral de alerta basado en los percentiles logró resultados similares o mejores que los otros algoritmos según el número de alertas que hubiera desencadenado. Para ese número de alertas, este algoritmo hubiera prevenido la mayor proporción de casos en comparación con los otros métodos. Con relación al momento óptimo de declarar la alerta, el algoritmo basado en los percentiles también tuvo el mejor rendimiento. El algoritmo basado en la pendiente del número de casos en la escala logarítmica ofreció resultados ligeramente mejores que el sistema de alertas aleatorias, pero fue el peor de los algoritmos estudiados. El algoritmo basado en la media de los casos semanales más su desviación es- 